Publ. RIMS, Kyoto Univ.

19 (1983), 519-547

\title{
Transformation Theory for Anti-Self-Dual Equations
}

By

Kimio UEno* and Yoshimasa NAKAMURA**

\begin{abstract}
An infinite-dimensional Lie algebra acting on solutions to the anti-self-dual equations on a four-dimensional Euclidean space is derived by means of the Riemann-Hilbert problem. Three types of Bäcklund transformations are considered in the framework of the Riemann-Hilbert problem.
\end{abstract}

\section{§1. Introduction}

In recent years remarkable progress has been made in studies on nonlinear field equations, and two possible approaches have been proposed. The first one is the symmetry theory which includes soliton theory and Bäcklund transformations. The second one has arisen from algebraic geometry.

In investigations of the symmetries, certain two-dimensional field equations have been found to admit infinite-dimensional Lie algebras: Kinnersley and Chitre [11] revealed that the Kac-Moody algebra $\mathfrak{G} \mathfrak{I}(2, \mathbb{R})$ $\otimes \boldsymbol{R}\left[\zeta, \zeta^{-1}\right]$ acts on solutions to the stationary axially symmetric graritational field equations. This Lie algebra originates in certain symmetries of the field equations. Hauser and Ernst [10] exponentiated all of these infinitesimal actions and constructed the transformation theory by means of the Riemann-Hilbert problem. Following their method, recently Ueno [15] has shown that the Kac-Moody algebras $\mathfrak{g} \mathfrak{u}(n) \otimes \mathbb{R}\left[\zeta, \zeta^{-1}\right]$, and $\mathfrak{g D}(n)$ $\otimes \mathbb{R}\left[\zeta, \zeta^{-1}\right]$ act on solutions to $\mathrm{SU}(\mathrm{n}), \mathrm{SO}(\mathrm{n})$ chiral fields, respectively.

Communicated by M. Sato, June 10, 198:. Revised September 20, 1982.

* Research Institute for Mathematical Sciences, Kyoto University, Kyoto 606, Japan. Current Adress: Department of Mathematics, Yoliohama City University, Yokohama 236, Japan

** Department of Applied Mathematics and Physics, Faculty of Engineering, Kyoto University, Kyoto 606, Japan 
We think it very important to extend these approaches to four-dimensional gauge fields.

Apart from these studies on symmetries, a great deal of progress has been made in the problem to find particular solutions to the gauge field equations, such as the instanton soluitons. Atiyah and Ward $[1,19]$ found that all the informations of $\mathrm{SU}(2)$ (anti-) self-dual Yang-Mills fields can be coded in a certain analytic vector bundle on $\boldsymbol{P}^{3}$. Their approach arises from Penrose's twistor theory, and is closely related to the RiemannHilbert problem.

In the soliton theory, Zakharov et al. $[3,21]$ made use of the Riemann-Hilbert problem to obtain the exact solutions to two-dimensional field equations. On the other hand, Dolan [7], by making use of the method of variations, discovered that the hidden symmetry algebras of chiral fields are the subalgebras $\mathfrak{g} \otimes \boldsymbol{R}[\zeta]$ of Kac-Moody algebras, and relevant discussions are given by Devchand and Fairlie [6], Chau et al. [4].

The purpose of this paper is to propose a transformation theory for the anti-self-dual Yang-Mills fields on a four-dimensional Euclidean space by means of the Riemann-Hilbert problem. The resulting infinitesimal transformation group is isomorphic to $\mathfrak{g l}(n, \boldsymbol{C}) \otimes C\left[\zeta, \zeta^{-1}, w_{1}, w_{2}\right]$. Furthermore we will discuss three types of Bäcklund transformations as applications of the Riemann-Hilbert transformations. They are different from known Bäcklund transformations (Prasad et al. [13], and Corrigan et al. [5]).

This paper is organized as follows: In Section 2, we will first review fundamental results on the anti-self-dual equation. The linear equations of Zakharov-Shabat's type associated with this equation will be also discussed. In Section 3, we will show the transformation theorem by using the Riemann-Hilbert problem, and will present the infinitesimal RiemannHilbert transformations. Also we will find the infinitesimal transformation group to be isomorphic to $\mathfrak{g l}(n, \boldsymbol{C}) \otimes C\left[\zeta, \zeta^{-1}, w_{1}, w_{2}\right]$. In the subsequent sections, Bäcklund transformations of three types will be considered as applications of the Riemann-Hilbert transformations. The first one gives 't Hooft's instanton solutions $(\S 4)$. The second one derives the Atiyah-Ward ansatz $(\S 5)$. The last one keeps the reality of gauge potentials and generalizes the transformation given by Belavin-Zakharov 
[2] (§6).

In the recent letter [16] we announced the results of the present paper. Here we will discuss more fully the derivation of those results and will report further developments.

The authors are deeply grateful to Professor Mikio Sato and Professor Masaki Kashiwara for many stimulating discussions and useful suggestions. One of the authors (Y.N.) also wish to thank Professor Mineo Ikeda for his encouragements.

\section{§ 2. Preliminaries and Linearization Problem for Anti-Self-Dual Equations}

First of all we discuss the anti-self-dual Yang-Mills fields on a fourdimensional Euclidean space with the coordinates $\left(x^{1}, x^{2}, x^{3}, x^{4}\right)$. Let $B_{\mu}$ be gauge potentials ( $n \lambda n$ matrices), and $F_{\mu \nu}$ gauge field strengths, $F_{\mu \nu}$ $=\partial_{\nu} B_{\mu}-\partial_{\mu} B_{\nu}-\left[B_{\mu}, B_{\nu}\right]$, for $\mu, \nu=1, \cdots, 4$. The anti-self-dual equations are a system of nonlinear equations defined by

$$
* F_{\mu \nu}=-F_{\mu \nu}
$$

where the symbol $*$ stands for the Hodge's star operator in $\mathbb{R}^{4}$. If some solutions $B_{\mu}$ are anti-hermitian and trace-free matrices (that is, they belong to the Lie algebra $\mathfrak{G} \mathfrak{u}(n))$, are referred to as $\mathfrak{g} \mathfrak{u}(n)$ gauge potentials. However, in this paper, we should rather think $B_{\mu}$ to be analytically continued into the complex space $\mathbb{C}^{4}$ where $x^{\mu}$ are complex. That is, $B_{\mu}$ are $n \times n$ complex matrices defined on $\mathbb{C}^{4}$. Let us now introduce complex variables $y, \bar{y}, z, \bar{z}$ through

$$
\begin{aligned}
& y=\frac{1}{2}\left(x^{1}+i x^{2}\right), \quad \bar{y}=\frac{1}{2}\left(x^{1}-i x^{2}\right), \\
& z=\frac{1}{2}\left(x^{3}+i x^{4}\right), \quad \bar{z}=\frac{1}{2}\left(x^{3}-i x^{4}\right) .
\end{aligned}
$$

Then the anti-self-dual equations reduce to three independent equations:

$$
\begin{aligned}
& F_{y z}=0=F_{\bar{y} \bar{z}}, \\
& F_{y \bar{y}} \mid F_{\bar{z} \bar{z}}=0,
\end{aligned}
$$

where $F_{y z}=\partial_{z} B_{y}-\partial_{y} B_{z}-\left[B_{y}, B_{z}\right]$ and $B_{y}=B_{1}-i B_{2}, B_{\bar{y}}=B_{1}+i B_{2}$ and so 
on. The equations (2.2) can be easily integrated as follows:

$$
\begin{array}{ll}
B_{y}=D^{-1} \partial_{y} D, & B_{\bar{y}}=\bar{D}^{-1} \partial_{\bar{y}} \bar{D} \\
B_{z}=D^{-1} \partial_{z} D, & B_{\bar{z}}=\bar{D}^{-1} \partial_{\bar{z}} \bar{D} .
\end{array}
$$

Here $D$ and $\bar{D}$ are $\operatorname{GL}(n, C)$-matrices. This idea was first presented by Yang [20]. Furthermore an important observation was made by Pohlmeyer [12] and Prasad et al. [13]. Let us define a $\mathrm{GL}(n, \boldsymbol{C})$-matrix $J$ by

$$
J=D \bar{D}^{-1}
$$

Then the second equation (2.3) leads to a single equation for $J$.

$$
\partial_{\bar{y}}\left(J^{-1} \partial_{y} J\right)+\partial_{\bar{z}}\left(J^{-1} \partial_{z^{\prime}} J\right)=0
$$

or, equivalently,

$$
\partial_{y}\left(\partial_{\bar{y}} J \cdot J^{-1}\right)+\partial_{z}\left(\partial_{\bar{z}} J \cdot J^{-1}\right)=0 .
$$

Conversely, for a solution $J$ to (2.6), we can reconstruct gauge potentials through (2.4) and (2.5). Thus the equation (2.6) is equivalent to the original equations (2.1). We simply call $(2.6)$ (or $\left.(2.6)^{\prime}\right)$ the anti-selfdual equation in this paper. We remark that it is easy to obtain an $\mathrm{SL}(n, \boldsymbol{C})$-solution to $(2.6)$, because we have the following lemma.

Lemma 2. 1. Suppose $J$ be a solution to (2.6). Then

$$
\tilde{J}=(\operatorname{det} J)^{-1 / n} J
$$

is an $\mathrm{SL}(n, \boldsymbol{C})$-solution to (2.6).

If $B_{\mu}$ are $\mathfrak{g} \mathfrak{u}(n)$ gauge potentials, we can take $\bar{D}$ to be $\left(D^{\dagger}\right)^{-1}$ for real $x_{\mu}$ ( $\dagger$ denotes the hermitian conjugate). Then $J$ is a positive definite, $\operatorname{SL}(n, \boldsymbol{C})$ hermitian matrix.

For a given solution to (2.6), other manners to reconstruct gauge fields are known. For example, let us consider the case of $n=2$. For $\mathrm{SL}(2, \boldsymbol{C})$-solution $J$, parametrizing $J$ as

$$
J=f^{-1}\left(\begin{array}{ll}
1 & -g \\
e & f^{2}-e g
\end{array}\right),
$$

we obtain gauge potentials 
(2.8)

$$
B_{\eta}=f^{-1}\left(\begin{array}{ll}
f_{y} & 0 \\
2 g_{\bar{z}} & -f_{y}
\end{array}\right), \quad B_{\bar{y}}=f^{-1}\left(\begin{array}{cc}
-f_{\bar{y}} & 2 c_{z} \\
0 & f_{\bar{y}}
\end{array}\right),
$$

$$
B_{z}=f^{-1}\left(\begin{array}{ll}
f_{z} & 0 \\
2 g_{\bar{y}} & -f_{z}
\end{array}\right), \quad B_{\bar{z}}=f^{-1}\left(\begin{array}{cc}
-f_{\bar{z}} & -2 e_{y} \\
0 & f_{\bar{z}}
\end{array}\right),
$$

where $f_{y}=\partial_{y} f$, and so on. The so-called 't Hooft's ansatz means to set $e=f=g$ in (2.8) ([14]).

Our transformation theory is established through a system of linear differential equations whose compatibility condition gives (2.6) or (2.6)'. The existence of such a system is insured by symmetries of the equation (2.6), and provides a starting point in our theory. Such a linear system for (2.6) has been firstly derived by Pohlmeyer [12]. However our system is a little different from his. First we introduce linear differential operator $D_{k}(k=1,2)$ through

$$
D_{1}=\zeta^{-1} \partial_{\bar{z}}+\partial_{y}, \quad D_{2}=\zeta^{-1} \partial_{\bar{y}}-\partial_{z},
$$

where $\zeta$ is a complex parameter. Set

$$
A_{1}=\partial_{\bar{z}} J \cdot J^{-1}, \quad A_{2}=\partial_{\bar{y}} J \cdot J^{-1} .
$$

Then the anti-self-dual equation $(2.6)^{\prime}$ reads

$$
\begin{aligned}
& \partial_{z} A_{1}+\partial_{y} A_{2}=0, \\
& \partial_{\bar{y}} A_{1}-\partial_{\bar{z}} A_{2}+\left[A_{1}, A_{2}\right]=0 .
\end{aligned}
$$

Bearing this fact in mind, we have the following proposition.

Proposition 2.2. (A) Suppose $A_{k}=A_{k}(y, \bar{y}, z, \bar{z}) \quad(k=1,2)$ be $n \times n$ matrices. The compatibility condition for the following linear sy'stem,

$$
D_{k} Y^{Y}(\zeta)=\zeta^{-1} A_{k} Y(\zeta), \quad(k=1,2),
$$

gives the anti-self-dual equation (2.11).

(B) If the linear system (2.12) is compatible, and $Y(\zeta)=$ $Y(y, \bar{y}, \approx, \bar{z} ; \zeta)$ is a fundamental solution matrix to (2.12), and holomorphic near $\zeta=0$, then a solution $J$ to the anti-self-dual equation (2.6) is given by

$$
J=Y(0)=\left.Y(y, \bar{y}, \approx, \bar{z} ; \zeta)\right|_{\zeta=0} .
$$


This proposition shows us that a transformation on a solution matrix to (2.12) induces one on a solution to $(2.6)$. This point is crucial in our theory. In fact, this consideration allows us to achieve the transformation theory by making use of the Riemann-Hilbert problem.

\section{§ 3. Riemann-Hilbert Transformations}

After the idea in the previous section, we consider the RiemannHilbert transformations and the infinitesimal Riemann-Hilbert transformations for the anti-self-dual equation. Let $Y(\zeta)$ be a fundamental solution matrix to (2.12), and $C$ an analytic curve in the complex $\zeta$-plane encircling the origin such that $Y(\zeta)$ is holomorphic in $C \cup C_{+}$. Here $C_{+}\left(C_{-}\right)$denotes the inside (resp. outside) of $C$.

Consider the Riemann-Hilbert problem to find matrices $X_{ \pm}(\zeta)$ holomorphic and invertible in $C \cup C$. satisfying

$$
X_{-}(\zeta)=Y_{+}(\zeta) H(\zeta), \quad \zeta \in C,
$$

and the normalization condition

$$
X_{-}(\infty)=1 .
$$

Here $H(\zeta)$ is defined by

$$
H(\zeta)=Y(\zeta) u(\zeta) Y(\zeta)^{-1},
$$

and $u(\zeta)$ is a $\operatorname{GL}(n, C)$-matrix analytic on $C$ such that $D_{k} u(\zeta)=0$ (k $=1,2)$. This implies that $u(\zeta)$ is a function in $\zeta, w_{1}, w_{2}$, where the variables $w_{1}, w_{2}$ are introduced through

$$
w_{1}=\bar{z}-\zeta^{-1} y, \quad w_{2}=\bar{y}+\zeta^{-1} z .
$$

Also notice that $\square u(\zeta)=0$, where $\square$ is the four-dimensional Laplacian, i.e. $\square=\partial_{y} \partial_{\bar{y}}+\partial_{z} \partial_{\bar{z}}$.

We assume that there exists a pair of fundamental solution matrices $X_{ \pm}(\zeta)$ of the above Riemann-Hilbert problem. We note that, when $u(\zeta)$ is very close to the unit matrix, this problem actually has the solutions.

Let us define

$$
\widetilde{Y}(\zeta)=\left\{\begin{array}{lll}
X_{+}(\zeta) Y(\zeta) & \text { in } & C_{+}, \\
X_{-}(\zeta) Y(\zeta) u(\zeta)^{-1} & \text { in } & C_{-},
\end{array}\right.
$$


and

$$
\begin{aligned}
& \tilde{A}_{1}=A_{1}+\partial_{y}\left(\left.\partial_{w} \mathrm{X}_{-}\right|_{w=0}\right), \\
& \tilde{A}_{2}=A_{2}-\partial_{z}\left(\left.\partial_{w} X_{-}\right|_{w=0}\right),
\end{aligned}
$$

where $w=\zeta^{-1}$. The reconstruction of the anti-self-dual solution is guaranteed by the following theorem.

Theorem 3.1. The matrix $\widetilde{Y}(\zeta)$ is a fundamental solution matrix to the equations

$$
D_{k} \widetilde{Y}(\zeta)=\zeta^{-1} \widetilde{A}_{k} \widetilde{Y}(\zeta) \quad(k=1,2) .
$$

Therefore $\tilde{J}=\widetilde{Y}(0)=\left.\widetilde{Y}(y, \bar{y}, z, \bar{z} ; \zeta)\right|_{\zeta=0}$ is a new solution to the antiself-dual equation.

Proof. Since $u(\zeta)$ is annihilated by the operators $D_{k}$, we have

$$
D_{k} X_{+} \cdot X_{+}^{-1}+\zeta^{-1} X_{+} A_{k} X_{+}^{-1}=D_{k} X_{-} \cdot X_{-}^{-1}+\zeta^{-1} X_{-} A_{k} X_{-}^{-1} .
$$

Set $\mathrm{X}(\zeta)=X_{ \pm}(\zeta)$ in $C_{ \pm}$. The above equations show us that

$$
D_{k} X(\zeta) \cdot X(\zeta)^{-1}+\zeta^{-1} X(\zeta) A_{k} X(\zeta)^{-1}
$$

are rational functions in $\zeta$ and have a simple zero at the infinity. Considering the Laurent expansions at the infinity, we have

$$
D_{k} X(\zeta)=\zeta^{-1} \tilde{A}_{k} X(\zeta)-\zeta^{-1} X(\zeta) A_{k} .
$$

Thus the theorem is proved.

Q.E.D.

Remark. If both $J$ and $u(\zeta)$ are $\operatorname{SL}(n, \mathbb{C})$-valued, so is $\tilde{J}$.

Next we give a rough sketch of constructing the representation of the infinitesimal Riemann-Hilbert transformations. The procedure of the proof is almost the same as in the references [10], [15]. See them for the details.

First we rewrite the problem $(3.1),(3.2)$ into the Fredholm integral equation ([10], [15])

$$
\widetilde{Y} \circ(1-K)=Y_{u^{-1}}
$$

where the integral operator $K$ is defined by 


$$
\begin{aligned}
(\Phi \circ K)(\zeta)= & \frac{1}{2 \pi i} \int_{c} \frac{d \zeta^{\prime}}{\zeta^{\prime}-\zeta} \Phi\left(\zeta^{\prime}\right)\left\{Y\left(\zeta^{\prime}\right)^{-1} Y(\zeta)\right. \\
& \left.-u\left(\zeta^{\prime}\right) Y\left(\zeta^{\prime}\right)^{-1} Y^{\prime}(\zeta) u(\zeta)^{-1}\right\}, \quad \zeta \in C .
\end{aligned}
$$

Solving (3.8) by mean of the Neumann expansion, we see that $Y(\zeta)$ is approximated as

$$
\begin{gathered}
\widetilde{Y}(\zeta) \sim\left(\left(Y u^{-1}\right) \circ(1+K)\right)(\zeta) \\
=Y(\zeta) u(\zeta)^{-1}+\frac{1}{2 \pi i} \int_{C} \frac{d \zeta^{\prime}}{\zeta^{\prime}-\zeta^{\prime}}\left\{Y\left(\zeta^{\prime}\right) u\left(\zeta^{\prime}\right)^{-1} Y\left(\zeta^{\prime}\right)^{-1} Y(\zeta)\right. \\
\left.\quad-Y(\zeta) u(\zeta)^{-1}\right\}, \quad \zeta \in C .
\end{gathered}
$$

Substituting $u(\zeta)=\exp v(\zeta)$ into the above equation, and neglecting the terms of higher order in $v(\zeta)$, we get

$$
\begin{aligned}
\widetilde{Y}(\zeta) \sim Y(\zeta) & -Y(\zeta) v(\zeta)+\frac{1}{2 \pi i} \int_{c} \frac{d \zeta^{\prime}}{\zeta^{\prime}-\zeta^{\prime}}\{Y(\zeta) v(\zeta) \\
& \left.-Y\left(\zeta^{\prime}\right) v\left(\zeta^{\prime}\right) Y\left(\zeta^{\prime}\right)^{-1} Y(\zeta)\right\}, \quad \zeta \in C
\end{aligned}
$$

Since the integrand in the above equation is analytic at $\zeta^{\prime}=\zeta$, the variable $\zeta$ can be analytically continued into $C_{+}$. Then we notice that

$$
\frac{1}{2 \pi i} \int_{c} \frac{d \zeta^{\prime}}{\zeta^{\prime}-\zeta} Y(\zeta) v(\zeta)=\frac{1}{2 \pi i} \int_{c} \frac{d \zeta^{\prime}}{\zeta^{\prime}-\zeta} Y\left(\zeta^{\prime}\right) v\left(\zeta^{\prime}\right), \quad \zeta \in C_{+} .
$$

To sum up, we obtain

$$
\begin{aligned}
\widetilde{Y}(\zeta) \sim Y(\zeta) & +\frac{1}{2 \pi i} \int_{C} d \zeta^{\prime} Y\left(\zeta^{\prime}\right) v\left(\zeta^{\prime}\right) G\left(\zeta, \zeta^{\prime}\right) \\
& -\frac{1}{2 \pi i} \int_{c} \frac{d \zeta^{\prime}}{\zeta^{\prime}-\zeta} Y\left(\zeta^{\prime}\right) v\left(\zeta^{\prime}\right), \quad \zeta \in C_{+},
\end{aligned}
$$

where $G\left(\zeta^{\prime}, \zeta\right)$ is defined by

$$
G\left(\zeta^{\prime}, \zeta\right)=\frac{-1}{\zeta^{\prime}-\zeta}\left\{Y\left(\zeta^{\prime}\right)^{-1} Y(\zeta)-1\right\}
$$

Let $Y^{(n)}$ and $G^{(m, n)}$ be the coefficients of the Taylor expansions of $Y(\zeta)$ and $G\left(\zeta^{\prime}, \zeta\right)$,

$$
Y(\zeta)=\sum_{n=0}^{\infty} Y^{Y(n)} \zeta^{n}, \quad G\left(\zeta^{\prime}, \zeta\right)=\sum_{m, n=0}^{\infty} G^{(m, n)} \zeta^{\prime m} \zeta^{n} .
$$


From (3.9), we see that, by the Riemann-Ilibert transformation $Y(\zeta)$ $\rightarrow \widetilde{Y}(\zeta)$, the coefficients $Y^{(n)}$ 's are infinitesimally transformed as follows;

$$
\begin{aligned}
Y^{(n)} \rightarrow Y^{(n)} & -\sum_{p=0}^{\infty} Y^{(p)} \boldsymbol{v}^{(p-n)} \\
& +\sum_{p, q=0}^{\infty} Y^{(p)} \boldsymbol{V}^{(p+q+1)} G^{(q, n)}
\end{aligned}
$$

where $v^{(p)}=\frac{1}{2 \pi i} \int_{C} d \zeta^{\prime} v\left(\zeta^{\prime}\right) \zeta^{\prime P-1}$. We call (3.10) the infinitesimal Riemann-Hilbert transformation ([15]).

Furthermore we set

$$
\begin{gathered}
\widehat{Y}(\zeta)=Y^{(0)-1} Y(\zeta)=\sum_{n=0}^{\infty} \widehat{Y}^{(n)} \zeta^{n} \\
\widehat{G}\left(\zeta^{\prime}, \zeta\right)=\frac{1}{\zeta^{\prime}-\zeta}\left\{\zeta^{\prime}-\zeta \widehat{Y}\left(\zeta^{\prime}\right)^{-1} \widehat{Y}(\zeta)\right\} \\
=\sum_{m, n=0}^{\infty} \widehat{G}^{(m, n)} \zeta^{\prime m} \zeta^{n}
\end{gathered}
$$

The following lemma is crucial in our procedure. Since the normalization point (the infinity in this case) is different from the one in [10], [15], we need this lemma.

Lemma 3.2. The coefficients $\widehat{G}^{(0, n)}$ are infinitesimally transformed by the Riemann-Hilbert transformation $Y(\zeta) \rightarrow \widetilde{Y}(\zeta)$ as follows;

$$
\widehat{G}^{(0, n)} \rightarrow \widehat{G}^{(0, n)}-\sum_{p=0}^{\infty} \widehat{G}^{(0, p)} V^{(p-n)}+\sum_{p, q=0}^{\infty} \widehat{G}^{(0, p)} V^{(p+q)} \widehat{G}^{(q, n)}
$$

Proof. By (3.10), we see that $\widehat{Y}^{(n)}$ are transformed as follows;

$$
\begin{aligned}
\widehat{Y}^{(n)} \rightarrow & \left\{Y^{(0)}-\sum_{p=0}^{\infty} Y^{(p)} \boldsymbol{V}^{(p)}+\sum_{p, q=0}^{\infty} Y^{(p)} \boldsymbol{v}^{(p+q+1)} G^{(q, 0)}\right\}^{-1} \\
& \times\left\{Y^{(n)}-\sum_{p=0}^{\infty} Y^{(p)} v^{(\rho-n)}+\sum_{p, q=0}^{\infty} Y^{(p)} v^{(p+q+1)} G^{(q, n)}\right\} \\
= & \left\{1+\sum_{p=0}^{\infty} \widehat{Y}^{(p)} \boldsymbol{V}^{(p)}-\sum_{p, q=0}^{\infty} \widehat{Y}^{(p)} \boldsymbol{v}^{(p+q+1)} G^{(q, n)}\right\} \\
& \times\left\{\widehat{Y}^{(n)}-\sum_{p=0}^{\infty} \widehat{Y}^{(p)} \boldsymbol{v}^{(p-n)}+\sum_{p, q=0}^{\infty} \widehat{Y}^{(p)} \boldsymbol{v}^{(p+q+1)} G^{(q, n)}\right\}
\end{aligned}
$$




$$
\begin{aligned}
=\widehat{Y}^{(n)} & -\sum_{p=0}^{\infty} \widehat{Y}^{(p)} v^{(p-n)}+\sum_{p, q=0}^{\infty} \widehat{Y}^{(p)} v^{(p+q+1)}\left\{G^{(q, n)}\right. \\
& \left.-G^{(q, 0)} \widehat{Y}^{(n)}\right\}+\sum_{p=0}^{\infty} \widehat{Y}^{(p)} v^{(p)} \widehat{Y}^{(n)} .
\end{aligned}
$$

Here we have neglected the terms of higher order in $v^{(p)}$. On the other hand, by the definition (3.11), we have

$$
\begin{aligned}
& \widehat{G}^{(0, n)}=\widehat{Y}^{(n)}, \text { for } n \geqq 0, \\
& \widehat{G}^{(m, n)}=G^{(m-1, n)}-G^{(m-1,0)} \widehat{Y}^{(n)}, \text { for } m \geqq 1, n \geqq 0 .
\end{aligned}
$$

Thus we obtain the infinitesimal transformation

$$
\begin{aligned}
\widehat{G}^{(0, n)} \rightarrow \widehat{G}^{(0, n)} & -\sum_{p=0}^{\infty} \widehat{G}^{(0, p)} \boldsymbol{v}^{(p-n)}+\sum_{p, q=0}^{\infty} \widehat{G}^{(0, p)} \boldsymbol{v}^{(p+q+1)} \widehat{G}^{(q+1, n)} \\
& +\sum_{p=0}^{\infty} \widehat{G}^{(0, p)} \boldsymbol{v}^{(p)} \widehat{G}^{(0, n)} \\
= & \widehat{G}^{(0, n)}-\sum_{p=0}^{\infty} \widehat{G}^{(0, p)} \boldsymbol{v}^{(p-n)}+\sum_{p, q=0}^{\infty} \widehat{G}^{(0, p)} \boldsymbol{v}^{(p+q)} \widehat{G}^{(q, n)} \text {. Q.E.D. }
\end{aligned}
$$

Especially let us consider an infinitesimal transformation associated with $v(\zeta)=v \zeta^{-k}$ where $v$ is a constant $\mathrm{gl}(n, C)$-matrix and $k$ is an positive integer. Following the method developed in [15], from (3.12), we get the generators for the infinitesimal Riemann-Hilbert transformations,

$$
\begin{gathered}
\widehat{G}^{(m, n)} \rightarrow \widehat{G}^{(m, n)}+v \widehat{G}^{(m+k, n)}-\widehat{G}^{(m, n+k)} v+\sum_{j=1}^{k} \widehat{G}^{(m, j)} v \widehat{G}^{(k-j, n)} \\
\quad \text { for } \quad k \geqq 0, m \geqq 0, n \geqq 1,
\end{gathered}
$$

and

$(3.13)_{-k} \quad \widehat{G}^{(m, n)} \rightarrow \widehat{G}^{(m, n)}+v \widehat{G}^{(m-k, n)}-\widehat{G}^{(m, n-k)} v+\sum_{j=0}^{k-1} \delta_{m, j} v \delta_{k-j, n}$

$$
\text { for } k \geqq 1, m \geqq 0, n \geqq 1 \text {, }
$$

where $\delta_{i j}$ is Kronecker's delta. In the right-hand side of $(3.13)_{-k}$, $\widehat{G}^{(m, n)}$ with negative indices is defined by

$$
\begin{aligned}
& \widehat{G}^{(m,-m)}=-\widehat{G}^{(-m, m)}=1 \quad \text { for } \quad m \geqq 1, \\
& \widehat{G}^{(m, n)}=0 \quad \text { for other negative indices. }
\end{aligned}
$$


We identify $r c^{\circ-k}$ with the infinitesimal transformation $(3.13)_{k}(k \in \mathbb{Z})$. In general we can naturally regard an infinitesimal transformation induced by $v(\zeta)=v \zeta^{-k} w_{1}^{k_{1}} w_{2}^{k_{2}}$ as a linear combination of (3.13) where $v$ is a constant matrix and $k, k_{1}, k_{2} \in \mathbb{Z}, k_{1}, k_{2} \geqq 0$. In other words,

$$
\begin{aligned}
& v \zeta^{-k} w_{1}^{k_{1}} w w_{2}^{k_{2}} \\
& \quad=v \zeta^{-k}\left\{\sum_{j_{1}=0}^{k_{1}}\left(\begin{array}{c}
k_{1} \\
j_{1}
\end{array}\right) \bar{y}^{j_{1}}\left(z \zeta^{-1}\right)^{k_{1}-j_{1}}\right\}\left\{\sum_{j_{2}=0}^{k_{2}}\left(\begin{array}{c}
k_{2} \\
j_{2}
\end{array}\right) \bar{z}^{j_{2}}\left(-y \zeta^{-1}\right)^{k_{2}-j_{2}}\right\}
\end{aligned}
$$

holds as an infinitesimal transformation.

Set

$$
\mathfrak{g}=\operatorname{span} \text { of }\left\{v \zeta^{-k} w_{1}^{k_{1}} w_{2}^{k_{2}} \mid v \in \mathfrak{g l}(n, \mathbb{C}), k, k_{1}, k_{2} \in \mathbb{Z}, k_{1}, k_{2} \geqq 0\right\} .
$$

Then we find $g$ to be equiped with a structure of a graded Lie algebra. We have the main theorem.

Theorem 3.3. As a graded Lie algebra, $g$ is isomorphic to

$$
g r(n, \mathbb{C}) \otimes C\left[\zeta, \zeta^{-1}, w_{1}, w_{2}\right]
$$

That is, the generators $v \zeta^{-k} w_{1}^{k_{1}} w_{2}^{k_{2}}, v^{\prime} \zeta^{-j_{1}} w_{1}^{j_{1}} w_{2}^{j_{2}}$ satisfy a commutation relation

$$
\left[v \zeta^{-k} w_{1}^{k_{1}} w_{2}^{k_{2}}, v^{\prime} \zeta^{-j} w_{1}^{j_{1}} w_{2}^{j_{2}}\right]=\left[v, v^{\prime}\right] \zeta^{-k-j_{1}} w_{1}^{k_{1}+j_{1}} w_{2}^{k_{2}+j_{2}}
$$

Here the bracket in the right-hand side is one of Lie algebra $\mathrm{gl}(n, C)$, and the bracket in the left-hand side is defined for two infinitesimal transformations.

The Lie algebra (3.14) is more complicated than the ones for the stationary axially symmetric gravitational field equations, or chiral fields. In fact, the Lie algebras (the infinitesimal transformation groups) for these equations are $\mathfrak{g y m}(2, \boldsymbol{R}) \otimes \boldsymbol{R}\left[\zeta, \zeta^{-1}\right]$, and $\mathfrak{g} \mathfrak{u}(n) \otimes \mathbb{R}\left[\zeta, \zeta^{-1}\right]$ (see $[10],[11],[15])$. Independently of the authors, Chau et al. [4] derived the infinitesimal transformation group $\mathfrak{g l}(n, \mathbb{C}) \otimes \mathbb{C}[\zeta]$ for the self-dual equation by use of the method of Dolan [7]. Forgács et al. also mentioned an invariance group for the self-dual equation in [8]. The link between our Riemann-Hilbert transformations and the method of Dolan and Chau et al. is discussed in [18]. 


\section{$\S 4$. Construction of 't Hooft's N-Instanton Solution}

In this section we consider the Riemann-Hilbert transformation (3.5) induced by

$$
\begin{aligned}
& u(\zeta)=1+\sum_{j=1}^{N}\left(\frac{a_{j}}{\zeta-\alpha_{j}}+\frac{b_{j}}{\zeta-\beta_{j}}+\frac{c_{j}}{\zeta-\zeta_{j}}\right) P, \\
& a_{j}=a_{j}^{\prime} /\left(\bar{z}-\bar{z}_{j}\right), \quad b_{j}=b_{j}^{\prime} /\left(\bar{y}-\bar{y}_{j}\right), \\
& a_{j}=\left(y-y_{j}\right) /\left(\bar{z}-\bar{z}_{j}\right), \quad \beta_{j}=-\left(z-z_{j}\right) /\left(\bar{y}-\bar{y}_{j}\right) .
\end{aligned}
$$

Here $a_{j}^{\prime}, b_{j}^{\prime}, c_{j}, y_{j}, z_{j}, \bar{y}_{j}, \bar{z}_{j}$ and $\zeta_{j}$ are complex constants and $P$ is a constant matrix such that $P^{2}=0$. We assume $\alpha_{j}, \beta_{j}$ and $\zeta_{j}$ to be mutually distinct and located in $C_{+}$. It is possible to obtain the solution $X_{ \pm}(\zeta)$ to the Riemann-Hilbert problem to (3.1) and (3.2) associated with (4.1) by an elementary operation of linear algebra. We call (4.1) an algebraic Riemann-Hilbert transformation (Bäcklund transformation).

We have the following theorem.

Theorem 4. 1. Let $Y(\zeta)$ be a fundamental solution matrix to (2.12). Then the solution matrix $X_{-}(\zeta)$ to the problem (3.1) and (3.2) with $u(\zeta),(4.1)$, takes the form

$$
X_{-}(\zeta)=1+\sum_{j=1}^{N}\left(\frac{R_{j}}{\zeta-\alpha_{j}}+\frac{S_{j}}{\zeta-\beta_{j}}+\frac{T_{j}}{\zeta-\zeta_{j}}\right)
$$

Here $R_{j}, S_{j}$ and $T_{j}$ are given by solving linear equations

$$
\begin{aligned}
\left(R_{1}, \cdots, R_{N}, S_{1}, \cdots, S_{N}, T_{1}, \cdots, T_{N}\right) W \\
=\left(a_{1} V\left(\alpha_{1}\right), \cdots, a_{N} V\left(\alpha_{N}\right), b_{1} V\left(\beta_{1}\right), \cdots,\right. \\
\left.b_{N} V\left(\beta_{N}\right), c_{1} V\left(\zeta_{1}\right), \cdots, c_{N} V\left(\zeta_{N}\right)\right),
\end{aligned}
$$

where $W=\left(W^{(k l)}\right)_{1 \leqq k, l \leqq 3}$ is a $3 n N \times 3 n N$ matrix and

$$
V\left(\alpha_{j}\right)=Y\left(\alpha_{j}\right) P Y\left(\alpha_{j}\right)^{-1}
$$

and so on. Each (ij)-minor block $(i, j=1, \cdots, N) W_{i j}^{(k l)}$ of $W^{(k l)}$ is

$$
W_{i j}^{(11)}=\frac{a_{j}}{\alpha_{i}-\alpha_{j}} V\left(\alpha_{j}\right),(i \neq j), W_{j j}^{(11)}=1-a_{j} \dot{V}\left(\alpha_{j}\right)
$$




$$
\begin{aligned}
& W_{i j}^{(12)}=\frac{a_{j}}{\beta_{i}-\alpha_{j}} V\left(\alpha_{j}\right), \quad W_{i j}^{(13)}=\frac{a_{j}}{\zeta_{i}-\alpha_{j}} V\left(\alpha_{j}\right), \\
& W_{i j}^{(21)}=\frac{b_{j}}{\alpha_{i}-\beta_{j}} V\left(\beta_{j}\right), \quad W_{i j}^{(22)}=\frac{b_{j}}{\beta_{i}-\beta_{j}} V\left(\beta_{j}\right),(i \neq j), \\
& W_{j j}^{(22)}=1-b_{j} \dot{V}\left(\beta_{j}\right), \quad W_{i j}^{(23)}=\frac{b_{j}}{\zeta_{i}-\beta_{j}} V\left(\zeta_{j}\right), \\
& W_{i j}^{(31)}=\frac{c_{j}}{\alpha_{i}-\zeta_{j}} V\left(\zeta_{j}\right), \quad W_{i j}^{(31)}=\frac{c_{j}}{\beta_{i}-\zeta_{j}} V\left(\zeta_{j}\right), \\
& W_{i j}^{(33)}=\frac{c_{j}}{\zeta_{i}-\zeta_{j}} V\left(\zeta_{j}\right),(i \neq j), \quad W_{j j}^{(33)}=1-c_{j} \dot{V}\left(\zeta_{j}\right),
\end{aligned}
$$

where $\dot{V}\left(\alpha_{j}\right)=\dot{Y}\left(\alpha_{j}\right) P Y\left(\alpha_{j}\right)^{-1}$ and so on.

Proof. Since

$$
\begin{aligned}
X_{+}(\zeta)= & X_{-}(\zeta) H(\zeta)^{-1} \\
= & \left\{1+\sum_{j=1}^{N}\left(\frac{R_{j}}{\zeta-\alpha_{j}}+\frac{S_{j}}{\zeta-\beta_{j}}+\frac{T_{j}}{\zeta-\zeta_{j}}\right)\right\} Y(\zeta) \\
& \times\left\{1-\sum_{j=1}^{N}\left(\frac{a_{j}}{\zeta-\alpha_{j}}+\frac{b_{j}}{\zeta-\beta_{j}}+\frac{c_{j}}{\zeta-\zeta_{j}}\right) P\right\} Y(\zeta)^{-1}
\end{aligned}
$$

must be holomorphic in $C_{+}$, the coefficients of $\left(\zeta-\alpha_{j}\right)^{-2},\left(\zeta-\beta_{j}\right)^{-2}$ and $\left(\zeta-\zeta_{j}\right)^{-2}$ vanish. So we obtain

$$
R_{j} V\left(\alpha_{j}\right)=0, \quad S_{j} V\left(\beta_{j}\right)=0, \quad T_{j} V\left(\zeta_{j}\right)=0 .
$$

Also the residue of $X_{+}(\zeta) Y(\zeta)$ at $\zeta=\alpha_{j}$ must vanish, so that we get

$$
\begin{aligned}
R_{j}(1 & \left.-a_{j} \dot{V}\left(\alpha_{j}\right)\right)+a_{j}^{\prime} \sum_{\substack{i=1 \\
i \neq j}}^{N} \frac{R_{i}}{\alpha_{i}-\alpha_{j}} V\left(\alpha_{j}\right) \\
& +a_{j}^{\prime} \sum_{i=1}^{N}\left(\frac{S_{j}}{\beta_{i}-\alpha_{j}}+\frac{T_{j}}{\zeta_{i}-\alpha_{j}}\right) V\left(\alpha_{j}\right)=a_{j}^{\prime} V\left(\alpha_{j}\right) .
\end{aligned}
$$

Similar equations as above are true for $\zeta=\beta_{j}, \zeta_{j}$. We observe that, since $P^{2}=0$, the first of (4.4) follows from (4.5). Equation (4.3) is equivalent to (4.5). This completes the proof.

Q.E.D.

As an application of Theorem 4.1, we construct 't Hooft's $N$-instanton solution of $\mathrm{SU}(2)$ anti-self-dual equations. We introduce $2 \times 2$ 
matrices $g^{(i)}(\zeta)$ through

$$
g^{(i)}(\zeta)=\left(\begin{array}{cc}
\frac{1+\phi^{(i+1)}(\zeta)}{1+\phi^{(i)}(\zeta)} & \frac{\phi^{(i)}(\zeta)-\phi^{(i+1)}(\zeta)}{1+\phi^{(i)}(\zeta)} \sum_{j=1}^{i} \frac{a_{j}}{\zeta-\alpha_{j}} \\
0 & 1
\end{array}\right)
$$

$0 \leqq i \leqq N-1$, where $\alpha_{j}$ and $a_{j}$ are defined as in (4.1), and

$$
\phi^{(0)}(\zeta)=0, \quad \phi^{(i)}=\frac{\zeta}{\zeta\left(\bar{y}-\bar{y}_{i}\right)+\left(z-z_{i}\right)},
$$

$1 \leqq i \leqq N$. Obviously $D_{k} g^{(i)}(\zeta)=0$ and $g^{(i)}(0)=1$. Set

$$
u^{(j)}(\zeta)=1+\frac{a_{j}^{\prime}}{\zeta-\alpha_{j}} P, \quad P=\left(\begin{array}{ll}
0 & 1 \\
0 & 0
\end{array}\right)
$$

$1 \leqq j \leqq N$. For a trivial solution $\widetilde{Y}^{(0)}=1$, we carry out successive transformations defined by

$$
\begin{aligned}
& Y^{(j)}(\zeta)=\widetilde{Y}^{(j)}(\zeta) g^{(j)}(\zeta), \\
& \widetilde{Y}^{(j+1)}(\zeta)=u^{(j+1)}(\zeta) \circ Y^{(j)}(\zeta),
\end{aligned}
$$

$0 \leqq j \leqq N-1$. Here the symbol $u^{(j+1)} \circ$ denotes the Bäcklund transformation induced by $u^{(j+1)}$. The solution $Y^{(j)}(\zeta)$ is obtained by multiplying $\widetilde{Y}^{(j)}(\zeta)$ into $g^{(j)}(\zeta)$.

We have the following proposition.

Proposition 4.2. The fundamental solution matrix $\widetilde{Y}^{(N)}(\zeta)$ gives a solution

$$
\begin{aligned}
\tilde{J}^{(N)} & =\widetilde{Y}^{(N)}(0) \\
& =\left(\begin{array}{cc}
1 & -\sum_{j=1}^{N} \frac{a_{j}^{\prime}}{\left(y-y_{j}\right)\left(\bar{y}-\bar{y}_{j}\right)+\left(z-z_{j}\right)\left(\bar{z}-\bar{z}_{j}\right)} \\
0 & 1
\end{array}\right) .
\end{aligned}
$$

Proof. The proof is done by induction. First we calculate $\widetilde{Y}^{(1)}(\zeta)$. By Theorem 4.1, the Bäcklund transformation induced by $u^{(1)}(\zeta)$ is

$$
\widetilde{Y}^{(1)}(\zeta)=X_{-}^{(1)}(\zeta) Y^{(0)}(\zeta) u^{(1)}(\zeta)^{-1},
$$

where $Y_{-}^{(1)}(\zeta)=1+R_{1} /\left(\zeta-\alpha_{1}\right)$, and 


$$
\begin{aligned}
R_{1}= & a_{1} Y^{(0)}\left(\alpha_{1}\right)\left(\begin{array}{ll}
0 & 1 \\
0 & 0
\end{array}\right) Y^{(0)}\left(\alpha_{1}\right)^{-1} \\
& \times\left\{1-a_{1} \dot{Y}^{(0)}\left(\alpha_{1}\right)\left(\begin{array}{ll}
0 & 1 \\
0 & 0
\end{array}\right) Y^{(0)}\left(\alpha_{1}\right)^{-1}\right\}^{-1} \\
= & a_{1}\left(\begin{array}{cc}
0 & 1+\phi^{(1)}\left(\alpha_{1}\right) \\
0 & 0
\end{array}\right) .
\end{aligned}
$$

Hence we obtain

$$
\widetilde{Y}^{(1)}(\zeta)=\left(\begin{array}{cc}
1+\phi^{(1)}(\zeta) & \frac{a_{1}}{\zeta-\alpha_{1}}\left(\phi^{(1)}\left(\alpha_{1}\right)-\phi^{(1)}(\zeta)\right) \\
0 & 1
\end{array}\right)
$$

We show that, in general,

(4.9) $\quad \widetilde{Y}^{(k)}(\zeta)$

$$
=\left(\begin{array}{cc}
1+\phi^{(k)}(\zeta) & \sum_{j=1}^{k} \frac{a_{j}}{\zeta-\alpha_{j}}\left(\phi^{(j)}\left(\alpha_{j}\right)-\phi^{(k)}(\zeta)\right) \\
0 & 1
\end{array}\right)
$$

by induction. Suppose that we have proved the $k$-th induction step. Multiplying $\widetilde{Y}^{(k)}(\zeta)$ into $g^{(k)}(\zeta)$ gives

$$
Y^{(k)}(\zeta)=\left(\begin{array}{cc}
1+\phi^{(k+1)}(\zeta) & \sum_{j=1}^{k} \frac{a_{j}}{\zeta-\alpha_{j}}\left(\phi^{(j)}\left(\alpha_{j}\right)-\phi^{(k+1)}(\zeta)\right) \\
0 & 1
\end{array}\right)
$$

By Theorem 4.1 and the assumption of induction, the Bäcklund transformation induced by $u^{(k+1)}(\zeta)$ is

$$
\widetilde{Y}^{(k+1)}(\zeta)=X_{-}^{(k+1)}(\zeta) Y^{(k)}(\zeta) u^{(k+1)}(\zeta)^{-1},
$$

where $X_{-}^{(k+1)}(\zeta)=1+R_{k+1} /\left(\zeta-\alpha_{k+1}\right)$, and

$$
R_{k+1}=a_{k+1}\left(\begin{array}{cc}
0 & 1+\phi^{(k+1)}\left(\alpha_{k+1}\right) \\
0 & 0
\end{array}\right)
$$

So we obtain

$$
\widetilde{Y}^{(k+1)}(\zeta)=\left(\begin{array}{cc}
1 & \frac{a_{k+1}}{\zeta-\alpha_{k+1}}\left(1+\phi^{(k+1)}\left(\alpha_{k+1}\right)\right) \\
0 & 1
\end{array}\right)
$$




$$
\begin{aligned}
& \times\left(\begin{array}{c}
1+\phi^{(k+1)}(\zeta) \quad \sum_{j=1}^{k} \frac{a_{j}}{\zeta-\alpha_{j}}\left(\phi^{(j)}\left(\alpha_{j}\right)-\phi^{(k+1)}(\zeta)\right) \\
0
\end{array}\right) \\
& \times\left(\begin{array}{cc}
1 & -\frac{a_{k+1}}{\zeta-\alpha_{k+1}} \\
0 & 1
\end{array}\right) \\
& =\left(\begin{array}{c}
1+\phi^{(k+1)}(\zeta) \sum_{j=1}^{k+1} \frac{a_{j}}{\zeta-\alpha_{j}}\left(\phi^{(j)}\left(\alpha_{j}\right)-\phi^{(k+1)}(\zeta)\right) \\
0
\end{array}\right) .
\end{aligned}
$$

Thus we have proved (4.9) for any $1 \leqq k \leqq N$. Substituting $\zeta=0$ into (4.9) $(k=N)$, we get $\tilde{J}^{(N)}$.

Q.E.D.

By a simple transformation

$$
J^{(N)}=\tilde{J}^{(N)}\left(\begin{array}{cc}
-1 & -1 \\
1 & 0
\end{array}\right)
$$

we derive 't Hooft $N$-instanton solution (see [14]). In the parametrization (2.7), it is represented as

$$
\mathcal{c}=f=g=-\left\{1+\sum_{j=1}^{N} \frac{a_{j}^{\prime}}{\left(y-y_{j}\right)\left(\bar{y}-\bar{y}_{j}\right)+\left(z-z_{j}\right)\left(\bar{z}-\bar{z}_{j}\right)}\right\}^{-1} .
$$

We observe that this solution has $5 N$ arbitrary parameters.

\section{§ 5. Atiyah-Ward Ansatz}

As an application of the Riemann-Hilbert transformation (3.5), we discuss the Atiyah-Ward ansatz [1], [19] studied by Corrigan et al. [5].

Let $Y(\zeta)$ be a fundamental solution matrix to (2.12) holomorphic near $\zeta=0$. For $Y(\zeta)$, we consider the Riemann-Hilbert transformation induced by

$$
u(\zeta)=\left(\begin{array}{cc}
\zeta & 0 \\
0 & \zeta^{-1}
\end{array}\right)=\zeta E_{1}+\zeta^{-1} E_{2} .
$$

Here $E_{1}=\left(\begin{array}{ll}1 & 0 \\ 0 & 0\end{array}\right), E_{2}=\left(\begin{array}{ll}0 & 0 \\ 0 & 1\end{array}\right)$. In this transformation, the resulting multiplier matrix $X_{-}(\zeta)$ takes the form 


$$
X_{-}(\zeta)=1+\zeta^{-1} T \text {. }
$$

Let $\widetilde{Y}(\zeta)$ be the resulting fundamental solution matrix,

$$
\widetilde{Y}(\zeta)=X_{-}(\zeta) Y(\zeta) u(\zeta)^{-1}
$$

Denote $Y_{n}$ and $\widetilde{Y}_{n}$ be the coefficient of the Taylor expansion of $Y(\zeta)$ and $\widetilde{Y}(\zeta)$, respectively,

$$
\begin{aligned}
& Y(\zeta)=\sum_{n=0}^{\infty} Y_{n} \zeta^{n}, \quad Y_{n}=\left(y_{n, i j}\right)_{1 \leqq i, j \leqq 2}, \\
& \widetilde{Y}(\zeta)=\sum_{n=0}^{\infty} \widetilde{Y}_{n} \zeta^{n}, \quad \widetilde{Y}_{n}=\left(\widetilde{y}_{n, i j}\right)_{1 \leqq i, j \leqq 2}
\end{aligned}
$$

Substituting (5.1), (5.2) and (5.4) into (5.3), we have

$$
\begin{aligned}
\widetilde{Y}(\zeta)= & \zeta^{-2} T Y_{0} E_{2}+\zeta^{-1}\left(T Y_{1} E_{2}+Y_{0}^{r} E_{2}\right)+Y_{1} E_{2}+T\left(Y_{0}^{\gamma} E_{1}+Y_{2} E_{2}\right) \\
& +\sum_{n+1}^{\infty}\left\{Y_{n-1} E_{1}+Y_{n+1} E_{2}+T\left(Y_{n} E_{1}+Y_{n+2} E_{2}\right)\right\} \zeta^{n} .
\end{aligned}
$$

The condition that the coefficients of $\zeta^{-2}$ and $\zeta^{-1}$ in the above Laurent expansion should vanish uniquely determines the matrix $T$.

Proposition 5. . (A): The malrix $T$ is given by

$$
T=\frac{1}{\Delta}\left(\begin{array}{cc}
y_{0,12} y_{0,22} & -\left(y_{0,12}\right)^{2} \\
\left(y_{0,22}\right)^{2} & -y_{0,12} y_{0,22}
\end{array}\right),
$$

where

$$
\Delta=y_{0,12} y_{1,22}-y_{1,12} y_{0,22} .
$$

(B): The coefficients $\widetilde{y}_{n, i j}$ are expressed as follows;

$$
\begin{aligned}
& \widetilde{y}_{0,11}=\Delta^{-1} y_{0,12}, \\
& \widetilde{y}_{n, 11}=y_{n-1,11}+\Delta^{-1} y_{0,12}\left(y_{0,22} y_{n, 11}-y_{0,12} y_{n, 21}\right), \\
& \widetilde{y}_{0,21}=\Delta^{-1} y_{0,22}, \\
& \widetilde{y}_{n, 21}=y_{n-1,21}+\Delta^{-1} y_{0,22}\left(y_{0,22} y_{n, 11}-y_{0,12}^{\prime} y_{n, 21}\right), \\
& \widetilde{y}_{n-1,12}=y_{n, 12}^{\prime}+\Delta^{-1} y_{0,12}\left(y_{0,22}^{\prime} y_{n+1,12}-y_{0,12} y_{n+1,2,2}\right), \\
& \widetilde{y}_{n-1,22}=y_{n, 22}+\Delta^{-1} y_{0,22}^{\prime}\left(y_{0,22}^{\prime} y_{n} \quad \therefore, 12-y_{0,12}^{\prime} y_{n+1,22}^{\prime}\right),
\end{aligned}
$$

for $n \geqq 1$. 
In the next stage, we will apply this proposition to study the AtiyahWard ansatz.

First we review the Atiyah-Ward ansatz. For the construction of instanton solutions of SU(2) (anti-) self-dual gauge fields, Atiyah and Ward [1], [19] considered an analytic vector bundle of rank 2 on $\boldsymbol{P}^{3}$ with a transition matrix

$$
g(y, \bar{y}, z, \bar{z} ; \zeta)=\left(\begin{array}{cc}
\zeta^{l} & \rho(\zeta) \\
0 & \zeta^{-l}
\end{array}\right)
$$

Here $l$ is an integer larger than 1 and $\rho(\zeta)=\rho(y, \bar{y}, z, \bar{z} ; \zeta)$ satisfies $D_{k} \rho(\zeta)=0,(k=1,2)$. Decomposing $g(\zeta)$ as

$$
g(\zeta)=Y^{(+)}(\zeta)^{-1} Y^{(-)}(\zeta),
$$

where $Y^{(+)}(\zeta)\left(Y^{(-)}(\zeta)\right)$ is a matrix function analytic away from $\zeta=0$ (resp. $\zeta=\infty$ ), Atiyah and Ward showed that $Y^{( \pm)}(\zeta)$ solve the following linear differential equations

$$
\begin{aligned}
& D_{1} Y^{( \pm)}(\zeta)=-\left(B_{y}+\zeta^{-1} B_{\bar{z}}\right) Y^{( \pm)}(\zeta) \\
& D_{2} Y^{( \pm)}(\zeta)=\left(B_{z}-\zeta^{-1} B_{\bar{y}}\right) Y^{( \pm)}(\zeta)
\end{aligned}
$$

The compatibility condition of these equations is equivalent to (2.3). Atiyah and Ward claimed that the decomposition (5.9) yields $l$-instanton solutions. The so-called Atiyah-Ward ansatz $\mathscr{A}_{l}$ means the (anti-) selfdual solution given by the transition matrix $g(\zeta)$ with $l$. It should be noted that our fundamental solution matrix $Y(\zeta)$ to $(2.12)$ is related to $Y^{( \pm)}(\zeta)$ by

$$
Y(\zeta)=Y^{(-)}(\infty)^{-1} Y^{(+)}(\zeta)
$$

Hence the solution $J_{l}$ to (2.6), corresponding to the ansatz $\mathscr{A}_{l}$, is given by

$$
J_{l}=Y^{(-)}(\infty)^{-1} Y^{(+)}(0)
$$

Taking this approach further, Corrigan, Fairlie, Yates and Goddard [5] succeeded in writing down the (anti-) self-dual solutions of the ansatz $\mathscr{A}_{l}$ by using the expansion

$$
\rho(\zeta)=\sum_{n=-\infty}^{\infty} \tau_{n} \zeta^{n}
$$


Since the condition $D_{k} \rho(\zeta)=0$ yields $\partial_{\bar{z}} \tau_{n}=-\partial_{y} \tau_{n-1}, \partial_{\bar{y}} \tau_{n}=\partial_{z} \tau_{n-1}$, each $\tau_{n}$ satisfies $\square \tau_{n}=0$. In our notation, their results are following: Let $J_{l}$ $=\left(J_{l, i j}\right)_{1 \leqq i, j \leqq 2}$. Then

$$
\begin{aligned}
& J_{l, 11}=(-)^{l}\left|\begin{array}{ccc}
\tau_{-l} & \cdots & \tau_{-1} \\
\vdots & & \vdots \\
\tau_{-1} & \cdots & \tau_{l-2}
\end{array}\right| /\left|\begin{array}{ccc}
\tau_{-l+1} & \cdots & \tau_{0} \\
\vdots & & \vdots \\
\tau_{0} & \cdots & \tau_{l-1}
\end{array}\right|, \\
& J_{l, 21}=(-)^{l+1}\left|\begin{array}{ccc}
\tau_{-l+2} & \cdots & \tau_{0} \\
\vdots & \vdots \\
\tau_{0} & \cdots & \tau_{l-2}
\end{array}\right| /\left|\begin{array}{ccc}
\tau_{-l+1} & \cdots & \tau_{0} \\
\vdots & \vdots \\
\tau_{0} & \cdots & \tau_{l-1}
\end{array}\right|, \\
& J_{l, 12}=(-)^{l+1}\left|\begin{array}{ccc}
\tau_{-l} & \cdots & \tau_{0} \\
\vdots & \vdots \\
\tau_{0} & \cdots & \tau_{l}
\end{array}\right| /\left|\begin{array}{ccc}
\tau_{-l+1} & \cdots & \tau_{0} \\
\vdots & \vdots \\
\tau_{0} & \cdots & \tau_{l-1}
\end{array}\right|, \\
& J_{l, 22}=(-)^{l}\left|\begin{array}{ccc}
\tau_{-l+2} & \cdots & \tau_{1} \\
\vdots & \vdots \\
\tau_{1} & \cdots & \tau_{l}
\end{array}\right| /\left|\begin{array}{ccc}
\tau_{-l+1} & \cdots & \tau_{0} \\
\vdots & \vdots \\
\tau_{0} & \cdots & \tau_{l-1}
\end{array}\right| .
\end{aligned}
$$

Let us shift the index of $J_{l, i j}$ as

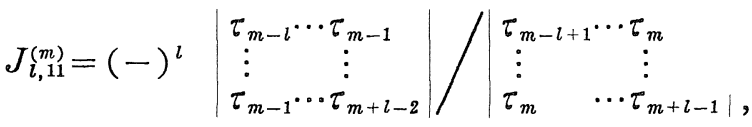

$$
\begin{aligned}
& J_{l, 21}^{(m)}=(-)^{l+1}\left|\begin{array}{ccc}
\tau_{m-l+2} & \cdots \tau_{m} \\
\vdots & \vdots \\
\tau_{m} & \cdots & \tau_{m+l-2}
\end{array}\right| /\left|\begin{array}{ccc}
\tau_{m-l+1} & \cdots \tau_{m} \\
\vdots & \vdots \\
\tau_{m} & \cdots & \tau_{m+l-1}
\end{array}\right|, \\
& J_{l, 12}^{(m)}=(-)^{l+1}\left|\begin{array}{ccc}
\tau_{m-l} & \cdots & \tau_{m} \\
\vdots & \vdots \\
\tau_{m} & \cdots & \tau_{m+l}
\end{array}\right| /\left|\begin{array}{ccc}
\tau_{m-l+1} & \cdots & \tau_{m} \\
\vdots & \vdots \\
\tau_{m} & \cdots & \tau_{m+l-1}
\end{array}\right|, \\
& J_{i, 22}^{(m)}=(-)^{l}\left|\begin{array}{cc}
\tau_{m-l+2} \cdots \tau_{m+1} \\
\vdots & \vdots \\
\tau_{m+1} & \cdots \tau_{m+l}
\end{array}\right| /\left|\begin{array}{cc}
\tau_{m-l+1} \cdots \tau_{m} \\
\vdots & \vdots \\
\tau_{m} & \cdots \tau_{m+l-1}
\end{array}\right| .
\end{aligned}
$$

Clearly $J_{l}^{(m)}=\left(J_{l, i j}^{(m)}\right)_{1 \leqq i, j \leqq 2}$ is also a solution to the (anti-) self-dual equation (2.6) and $J_{l}^{(0)}=J_{l}$. We call $J_{l}^{(m)}$ ansatz $\mathscr{A}_{l}^{(m)}$. Corrigan er al. obtained $J_{l}$ by applying successively simple Bäcklund transformations on $J_{1}([5], \S 4)$. We will derive the solution $J_{l}^{(m)}$ by using the RiemannHilbert transformation induced by $u(\zeta),(5.1)$, independently of their method.

Let $Y^{r(0)}(\zeta)$ be a fundamental solution matrix to $(2.12)$ corresponding to the ansatz $\mathcal{A}_{1}^{(0)}$, and set

$$
Y^{(l+1)}(\zeta)=u(\zeta) \circ Y^{(l)}(\zeta)
$$


$l \geqq 0$, recursively. Here $u(\zeta) \circ$ means the Riemann-Hilbert transformation induced by $u(\zeta)$.

We now give the solution $Y^{(0)}(\zeta)$.

Lemma 5.2. ([5]) Let $Y_{n}^{(0)}=\left(y_{n, i j}^{(0)}\right)_{1 \leqq i, j \leqq 2}$ be the coefficient of the Taylor expansion of $Y^{(0)}$ :

$$
Y^{(0)}(\zeta)=\sum_{n=0}^{\infty} Y_{n}^{(0)} \zeta^{n}
$$

Then $Y_{n}^{(0)}$ is expressed by using $\tau_{k}$ 's as

$$
\left\{\begin{array}{l}
y_{0,11}^{(0)}=-\tau_{-1} / \tau_{0}, \quad y_{1,11}^{(0)}=1, \quad y_{n, 11}^{(0)}=0, \text { for } n \geqq 2, \\
y_{0,21}^{(0)}=1 / \tau_{0}, \quad y_{n, 21}^{(0)}=0, \quad \text { for } n \geqq 1, \\
y_{n, 12}^{(0)}=\left|\begin{array}{ll}
\tau_{-1} & \tau_{n} \\
\tau_{0} & \tau_{n+1}
\end{array}\right|: \tau_{0}, \quad y_{n, 22}^{(0)}=-\tau_{n+1} / \tau_{0} .
\end{array}\right.
$$

This lemma is proved by direct calculation. For the details, the reader should refer to [5]. In general, the coefficients of the Taylor expansion of $Y^{(l)}$ can be expressed as ratio of determinants. We have the main theorem in this section.

Theorem 5.3. Let $Y_{n}^{(l)}=\left(y_{n, i j}^{(l)}\right)_{1 \leqq i, j \leqq 2}$ be the coefficients of the Taylor expansion of $Y^{(l)}(\zeta)$,

$$
Y^{(l)}(\zeta)=\sum_{n=0}^{\infty} Y_{n}^{(l)} \zeta^{n}
$$

Then $Y_{n}^{(l)}$ is written down through $\tau_{k}$ 's as follows:

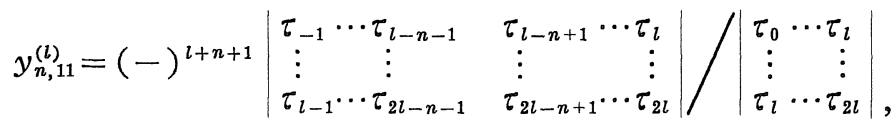

$$
\begin{aligned}
& (0 \leqq n \leqq l+1) \text {, } \\
& y_{n, 11}^{(l)}=0, \quad(l+2 \leqq n),
\end{aligned}
$$

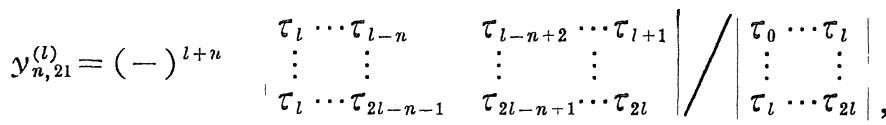

$$
\begin{aligned}
& (0 \leqq n \leqq l),
\end{aligned}
$$




$$
y_{n, 21}^{(l)}=0, \quad(l+1 \leqq n),
$$

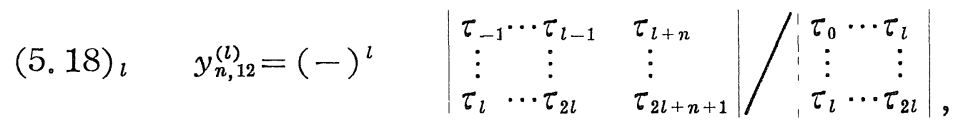

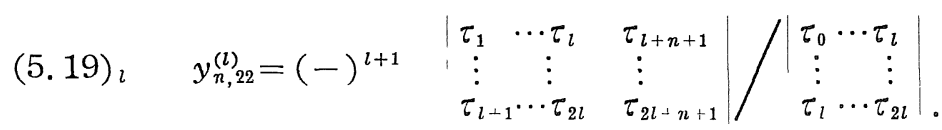

Set $\Delta_{l}=y_{0,12}^{(l)} y_{1,22}^{(l)}-y_{1,12}^{(l)} y_{0,22}^{(l)}$, then

$(5.20)_{l} \quad \Delta_{l}=\left|\begin{array}{ccc}\tau_{0} & \cdots & \tau_{l+1} \\ \vdots & \vdots \\ \tau_{l+1} & \cdots & \tau_{2 l+2}\end{array}\right| /\left|\begin{array}{ccc}\tau_{0} & \cdots & \tau_{l} \\ \vdots & & \vdots \\ \tau_{l} & \cdots & \tau_{2 l}\end{array}\right|$

Before proceeding to the proof, we prepare the following lemmas in linear algebra.

Lemma 5.4. ([9], p. 31) Let $M$ be an $n \times n$ matrix and $\Delta\left(\begin{array}{l}i_{1} \cdots i_{r} \\ k_{1} \cdots k_{r}\end{array}\right)$ a minor determinant defined by striking out the $i_{1}-t h, \cdots$, the $i_{r}$-th row's and the $k_{1}-t h, \cdots$, the $k_{r}$-th columns of $M$. Then

$$
|M| \cdot \Delta\left(\begin{array}{l}
i j \\
k l
\end{array}\right)=\Delta\left(\begin{array}{l}
i \\
k
\end{array}\right) \Delta\left(\begin{array}{l}
j \\
l
\end{array}\right)-\Delta\left(\begin{array}{l}
i \\
l
\end{array}\right) \Delta\left(\begin{array}{l}
j \\
k
\end{array}\right)
$$

holds for $i<j, k<l$.

From this lemma, we have

Lemma 5.5. The following identity holds:

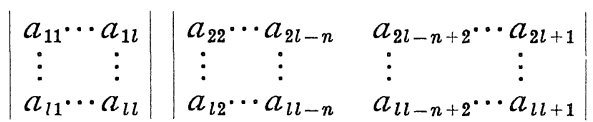

$$
\begin{aligned}
& +\left|\begin{array}{ll}
a_{12} \cdots & a_{1 l+1} \\
\vdots & \vdots \\
a_{l 2} \cdots & a_{l l+1}
\end{array}\right|\left|\begin{array}{cccc}
a_{21} \cdots & a_{2 l-n} & a_{2 l-n+2} \cdots & a_{2 l} \\
\vdots & \vdots & \vdots & \vdots \\
a_{l 1} \cdots & a_{l l-n} & a_{l l-n+2} \cdots & a_{l l}
\end{array}\right|
\end{aligned}
$$

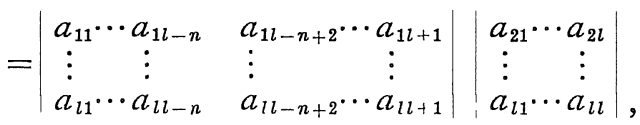

$$
\begin{aligned}
& 1 \leqq n \leqq l+1 \text {. }
\end{aligned}
$$


Proof of Theorem 5.3. We only show (5.18) ${ }_{l}$ and $(5.20)_{l}$ because the other equations can be proved in the same way. The proof is done by induction.

First let us derive $(5.20)_{l}$. Suppose the $l$-th induction step has been accomplished. Substituting $(5.18)_{l}$ and $(5.19)_{l}$ into the definition of $\Delta_{l}$, we have

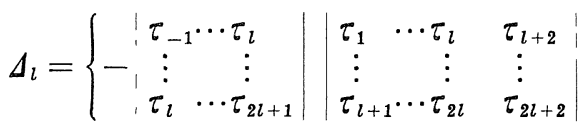

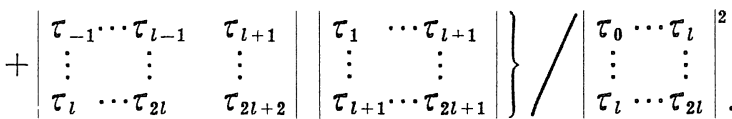

Then by Lemma 5.5 , we prove $(5.20)_{l}$. It should be noted that a general formula

$$
\begin{aligned}
& y_{0,12}^{(l)} y_{n, 22}^{(l)}-y_{n, 12}^{(l)} y_{0,22}^{(l)} \\
& \quad=\left|\begin{array}{cccc}
\tau_{0} & \cdots & \tau_{l} & \tau_{l+n} \\
\cdots & \vdots & \vdots \\
\tau_{l+1} & \cdots & \tau_{2 l+1} & \tau_{2 l+n+1}
\end{array}\right| /\left|\begin{array}{ccc}
\tau_{0} & \cdots & \tau_{l} \\
\vdots & \vdots \\
\tau_{l} & \cdots & \tau_{2 l}
\end{array}\right|
\end{aligned}
$$

can be derived by a similar method.

Next we proceed to the proof of $(5.18)_{l+1}$. By Proposition 5.1, $y_{n, 12}^{(l+1)}$ is given as follows:

$$
y_{n, 12}^{(l+1)}=y_{n+1,12}^{(l)}-\Delta_{l}^{-1} y_{0,12}^{(l)}\left(y_{0,12}^{(l)} y_{n+2,22}^{(l)}-y_{n+2,12}^{(l)} y_{0,22}^{(l)}\right) .
$$

Substituting (5.18) ${ }_{\iota}$ and (5.23) into the above equation, we get

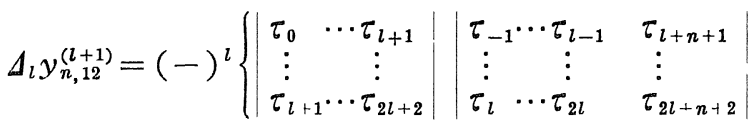

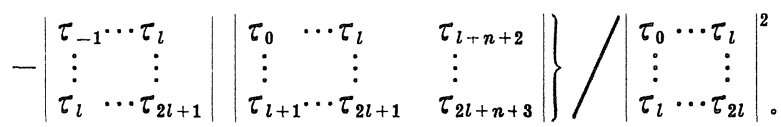

Then by Lemma 5.4 , we prove $(5.18)_{l+1}$.

Q.E.D.

Let us define

$$
J^{(l)}=Y_{0}^{(l)}=Y^{(l)}(0) \text {. }
$$

Comparing $J_{l}^{(m)},(5.14)$, with $(5.16)_{l^{-}}(5.19)_{l}$, we see $J^{(l)}$ is equivalent 
to $J_{l}^{(l)}$ of (5.14). That is to say, our Riemann-Hilbert transformation (5.1) produces the ansatz $\mathscr{A}_{l+1}^{(l)}$ from the ansatz $\mathscr{A}_{1}^{(0)}$.

\section{§6. Reality Conditions}

As we mentioned in Section 2, for $\mathfrak{g} \mathfrak{u}(n)$ gauge potentials, a matrix $J$ is required to be positive definite $\operatorname{SL}(n, \mathbb{C})$ hermitian on the real sector. Therefore, it is important to establish solution generating transformations which keep the reality conditions. In this section, we discuss a degenerate Riemann-Hilbert transformation preserving the reality. Belavin and Zakharov [2] studied such degenerate transformation to construct 't Hooft's instanton solutions. Their transformations were formulated in the $\operatorname{SL}(2, \mathbb{C})$ case only. In what follows, we will generalize their approach to the $\operatorname{SL}(n, \mathbb{C})$ case. We remark that our transformations do not necessarily retain the positivity of solutions.

Let $Y(\zeta)$ be a fundamental solution matrix to (2.12) and $J=Y(0)$ be hermitian on a real Euclidean space defined by $\bar{y}=y^{*}$ and $\bar{z}=z^{*}$ (* stands for the complex conjugate). Henceforth we will work in the real Euclidean space.

The degenerate Riemann-Hilbert transformaion takes the form

$$
\widetilde{Y}(\zeta)=X(\zeta) Y(\zeta)
$$

where $X(\zeta)=X\left(y, y^{*}, z,, z^{*} ; \zeta\right)$ is rational in $\zeta$ and is required to satisfy a normalization condition

$$
X(\infty)=X\left(y, y^{*}, z, z^{*} ; \zeta\right)_{\zeta=\infty}=1 .
$$

This transformation is based upon the idea proposed by Belinsky and Zakharov [3] to study the gravitational field equations. We have the following lemma.

Lemma 6. 1. Set $\tilde{J}=\widetilde{Y}(0)$. If $J$ is hermitian and $X(\zeta)$. $J X\left(-\zeta^{*-1}\right)^{+}$is independent of $\zeta$, then

$$
\tilde{J}=X(\zeta) J X\left(-\zeta^{*-1}\right)^{\dagger}, \quad \tilde{J}=\tilde{J}^{\dagger} .
$$

Here $\dagger$ denotes the hermitian conjugate. 
Proof. From the assumption, we have

$$
X(\zeta) J X\left(-\zeta^{*-1}\right)^{\dagger}=X(0) J X(\infty)^{\dagger} .
$$

Since $X(\infty)=1$ and $\tilde{J}=X(0) J$, we get the first assertion of (6.3). Similarly we obtain

$$
X(\zeta) J X\left(-\zeta^{*-1}\right)^{\dagger}=X(\infty) J X(0)^{\dagger}=\hat{J}^{\dagger} .
$$

This completes the proof.

Q.E.D.

Let us consider the degenerate Riemann-Hilbert transformation given by

$$
\begin{aligned}
X(\zeta)= & +\sum_{j=1}^{N_{1}} \frac{K_{j}}{\zeta-\zeta_{j}}+\sum_{j=1}^{N_{2}} \frac{L_{j}}{\zeta z^{*}-y-\zeta z_{j}^{*}+y_{j}} \\
& +\sum_{j=1}^{N_{3}} \frac{M_{j}}{\zeta y^{*}+z-\zeta y_{j+N_{2}}^{*}-z_{j+N_{2}}}, \\
X(\zeta)^{-1}= & 1+\sum_{j=1}^{N_{1}} \frac{K_{j}^{\prime}}{\zeta+\zeta_{j}^{*-1}}+\sum_{j=1}^{N_{2}} \frac{L_{j}^{\prime}}{\zeta y^{*}+z-\zeta y_{j}^{*}-z_{j}} \\
& +\sum_{j=1}^{N_{s}} \frac{M_{j}^{\prime}}{\zeta z^{*}-y-\zeta z_{j+N_{2}}^{*}+y_{j+N_{2}}},
\end{aligned}
$$

where $\zeta_{j}, y_{j}$ and $z_{j}$ are constants. Let us set

$$
\alpha_{j}=\frac{y-y_{j}}{z^{*}-z_{j}^{*}}, \quad \beta_{j}=-\frac{z-z_{j+N_{2}}}{y^{*}-y_{j+N_{2}}^{*}} .
$$

We assume that $\zeta_{j},-\zeta_{j}^{*-1}, \alpha_{j},-\alpha_{j}^{*-1}, \beta_{j}$ and $-\beta_{j}^{*-1}$ are mutually distinct. If $X(\zeta) J X\left(-\zeta^{*-1}\right)^{\dagger}$ and

$$
D_{k} \widetilde{Y}(\zeta) \cdot \widetilde{Y}(\zeta)^{-1}=D_{k} X(\zeta) \cdot X(\zeta)^{-1}+\zeta^{-1} X(\zeta) A_{k} X(\zeta)^{-1}
$$

are holomorphic at the poles $\zeta_{j},-\zeta_{j}^{*-1}, \alpha_{j},-\alpha_{j}^{*-1}, \beta_{j}$ and $-\beta_{j}^{*-1}$ (or equivalently, they are independent of $\zeta$ ), then the transformation gives a hermitian solution to the anti-self-dual equation (2.6). Since the residues $K_{j} X\left(\zeta_{j}\right)^{-1}, L_{j} X\left(\alpha_{j}\right)^{-1}, M_{j} X\left(\beta_{j}\right)^{-1}$ of $X(\zeta) X(\zeta)^{-1}$ at $\zeta=\zeta_{j}, \alpha_{j}, \beta_{j}$ must vanish, $K_{j}, L_{j}$ and $M_{j}$ are degenerate matrices. We also assume that these matrices are of rank 1 , that is,

$$
K_{j}=S_{j}{ }^{t} P_{j}, \quad L_{j}=T_{j}{ }^{t} Q_{j}, \quad M_{j}=U_{j}{ }^{t} R_{j},
$$

where $P_{j}, Q_{j}, R_{j}, S_{j}, T_{j}$ and $U_{j}$ are $n$-dimensional column vectors and 
${ }^{\prime} P_{j}$ denotes the transposed vector of $P_{i}$. We remark that

$$
{ }^{t} P_{j} \mathrm{X}\left(\zeta_{j}\right)^{-1}={ }^{t} Q_{j \alpha} \mathrm{X}\left(\zeta_{j}\right)^{-1}={ }^{t} R_{i} \mathrm{X}\left(\zeta_{j}\right)^{-1}=0 .
$$

We have the following theorem.

Theorem 6.2. Let us define $P_{j}, Q_{j}, R_{j}, S_{j}, T_{j}$ and $U_{j}$ by

$$
\begin{aligned}
& { }^{t} P_{j}={ }^{t} p_{j} Y\left(\zeta_{j}\right)^{-1}, \quad{ }^{t} Q_{j}={ }^{t} q_{j} Y\left(\alpha_{j}\right)^{-1}, \\
& { }^{t} R_{j}={ }^{t} r_{j} Y\left(\beta_{j}\right)^{-1}, \\
& \left(S_{1}, \cdots, S_{N_{1}}, T_{1}, \cdots, T_{N_{2}}, U_{1}, \cdots, U_{N_{3}}\right) Z \\
& \quad=\left(J P_{1}^{*}, \cdots, J P_{N_{1}}^{*}, J Q_{1}^{*}, \cdots, J Q_{N_{2}}^{*}, J R_{1}^{*}, \cdots, J R_{N_{3}}^{*}\right) .
\end{aligned}
$$

Here $p_{j}, q_{j}$ and $r_{j}$ are column vectors such that

$$
\begin{aligned}
& D_{k\left(\xi_{j}\right)} p_{j}=D_{k\left(\alpha_{j}\right)} q_{j}=D_{k\left(\beta_{j}\right)} r_{j}=0 \\
& D_{1(\xi)}=\xi^{-1} \partial_{\bar{z}}+\partial_{y}, \quad D_{2(\xi)}=\xi^{-1} \partial_{\bar{y}}-\partial_{z} .
\end{aligned}
$$

$Z=\left(Z^{(i j)}\right)_{1 \leqq i, j \leqq 3}$ is a $3\left(N_{1}+N_{2}+N_{3}\right) \times 3\left(N_{1}+N_{2}+N_{3}\right)$ matrix whose $(i, j)$-block $Z^{(i j)}=\left(Z_{k l}^{(i j)}\right)_{1 \leqq k \leqq N_{i}}, 1 \leqq l \leqq N_{j}$ is given by

$$
\begin{aligned}
& Z_{k l}^{(11)}=\frac{{ }^{t} P_{k} J P_{l}^{*}}{\zeta_{l}^{*-1}+\zeta_{k}}, \quad Z_{k l}^{(12)}=\frac{{ }^{t} P_{k} J Q_{l}^{*}}{\alpha_{l}^{*-1}+\zeta_{k}}, \\
& Z_{k l}^{(13)}=\frac{{ }^{t} P_{k} J R_{l}^{*}}{\beta_{l}^{*-1}+\zeta_{k}}, \quad Z_{k l}^{(21)}=\frac{{ }^{t} Q_{k} J P_{l}^{*}}{\left(z-z_{k}^{*}\right)\left(\zeta_{l}^{*-1}+\alpha_{k}\right)}, \\
& Z_{k l}^{(22)}=\frac{{ }^{t} Q_{k} J R_{l}^{*}}{\left(z-z_{k}^{*}\right)\left(\alpha_{l}^{*-1}+\alpha_{k}\right)}, \\
& Z_{k l}^{(23)}=\frac{{ }^{t} Q_{k} J R_{l}^{*}}{\left(z-z_{k}^{*}\right)\left(\beta_{l}^{*-1}+\alpha_{k}\right)}, \\
& Z_{k l}^{(31)}=\frac{{ }^{t} R_{k} J P_{l}^{*}}{\left(y-y_{N_{2}+k}^{*}\right)\left(\zeta_{l}^{*-1}+\beta_{k}\right)}, \\
& Z_{k l}^{(32)}=\frac{{ }^{t} R_{k} J Q_{l}^{*}}{\left(y-y_{N_{2}+k}^{*}\right)\left(\alpha_{l}^{*-1}+\beta_{k}\right)}, \\
& Z_{k l}^{(33)}=\frac{{ }^{t} R_{k} J R_{l}^{*}}{\left(y-y_{N_{2}+k}^{*}\right)\left(\beta_{l}^{*-1}+\beta_{k}\right)},
\end{aligned}
$$

where $P_{j}^{*}$ denotes the complex conjugate vector of $P_{j} . \quad$ Then $\tilde{J}=X(0) J$ 
is a hermitian solution to the anti-self-dual equation.

Proof. To begin with, let us consider the condition that

$$
D_{k} X(\zeta) \cdot X(\zeta)^{-1}+\zeta^{-1} X(\zeta) A_{k} X(\zeta)^{-1}
$$

is holomorphic at $\zeta=\zeta_{j}$. This requirement is equivalent to

$$
D_{k\left(\zeta_{j}\right)} K_{j} \cdot X\left(\zeta_{j}\right)^{-1}+\zeta_{j}^{-1} K_{j} A_{k} X\left(\zeta_{j}\right)^{-1}=0 .
$$

Notice that, by (6.6),

$$
D_{k\left(\zeta_{j}\right)} K_{j} \cdot X\left(\zeta_{j}\right)^{-1}=S_{j}\left(D_{k\left(\zeta_{j}\right)}^{t} P_{j}\right) X\left(\zeta_{j}\right)^{-1} .
$$

We see that the sufficient condition for $(6.11)$ is

$$
D_{k\left(\zeta_{j}\right)}{ }^{t} P_{j}+\zeta_{j}^{-1}{ }^{t} P_{j} A_{k}=0 \text {. }
$$

On the other hand,

$$
D_{k\left(\zeta_{j}\right)} Y\left(\zeta_{j}\right)^{-1}+\zeta_{j}^{-1} Y\left(\zeta_{j}\right)^{-1} A_{k}=0 .
$$

Comparing (6.12) with (6.13), we obtain the first equation in (6.7). The second and the third equations follow from the regularity at $\zeta=\alpha_{j}$, $\beta_{j}$ of $D_{k} X \cdot X^{-1}+\zeta^{-1} X A_{k} X^{-1}$.

Next we consider (6.8). The requirement that $X(\zeta) J X\left(-\zeta^{*-1}\right)^{\dagger}$ is holomorphic at $\zeta=-\zeta_{j}^{*-1},-\alpha_{j}^{*-1},-\beta_{j}^{*-1}$ implies

$$
\begin{aligned}
& \operatorname{Res}_{\zeta=-\zeta_{j}^{*-1}} X(\zeta) J X\left(-\zeta^{*-1}\right)^{\dagger}=X\left(-\zeta_{j}^{*-1}\right) J K_{j}^{\dagger}=0, \\
& X\left(-\alpha_{j}^{*-1}\right) J L_{j}^{\dagger}=X\left(-\beta_{j}^{*-1}\right) J M_{j}^{\dagger}=0 .
\end{aligned}
$$

When (6.14) holds, $X(\zeta) J X\left(-\zeta^{*-1}\right)^{\prime}$ is consequently holomorphic at $\zeta$ $=\zeta_{j}, \alpha_{j}, \beta_{j}$ because $J$ is hermitian. Since the first equation in (6.14) yields

$$
\begin{aligned}
J P_{j}^{*}= & \sum_{k=1}^{N_{1}} \frac{{ }^{t} P_{k} J P_{j}^{*}}{\zeta_{j}^{*-1}+\zeta_{k}} S_{k}+\sum_{k=1}^{N_{2}} \frac{{ }^{t} Q_{k} J P_{j}^{*}}{\left(z^{*}-z_{k}^{*}\right)\left(\zeta_{j}^{*-1}+\alpha_{k}\right)} T_{k} \\
& +\sum_{k=1}^{N_{3}} \frac{{ }^{t} R_{k} J P_{j}^{*}}{\left(y^{*}-y_{N_{2}+k}^{*}\right)\left(\zeta_{j}^{*-1}+\beta_{k}\right)} U_{k},
\end{aligned}
$$

and the second and the third ones in (6.14) also derive similar equations as above, we obtain (6.8) and (6.10). Thus Lemma 6.1 and (6.8) and (6.10) guarantee that $\tilde{J}$ is hermitian. 
Finally we show that $\tilde{J}$ is actually a solution to the anti-self-dual equation, in other words.

$$
\begin{aligned}
& D_{k} X(\zeta) \cdot X(\zeta)^{-1}+\zeta^{-1} X(\zeta) \Lambda_{k} X(\zeta)^{-1}=\zeta^{-1} \tilde{A}_{k}, \\
& \tilde{A}_{1}=\partial_{\bar{z}} \tilde{J} \cdot \tilde{J}^{-1}, \quad \tilde{A}_{2}=\partial_{\bar{y}} \tilde{J} \cdot \tilde{J}^{-1} .
\end{aligned}
$$

To this end, we prove the following lemma.

Lemma 6.3. Lel us sel

$$
B_{k}(\zeta)=D_{k} \mathrm{X}(\zeta) \cdot \mathrm{X}(\zeta)^{-1}+\zeta^{-1} X(\zeta) A_{k} \mathrm{X}(\zeta)^{-1}
$$

$k=1,2$. If $B_{k}(\zeta)$ are holomorphic at $\zeta=\zeta_{j}, \alpha_{j}, \beta_{j}$ and $\mathrm{X}(\zeta)$. $J X\left(-\zeta^{*-1}\right)^{\dagger}$ is holomorphic al $\zeta=\zeta_{j},-\zeta_{j}^{*-1}, \alpha_{j},-\alpha_{j}^{*-1}, \beta_{j},-\beta_{j}^{*-1}$, then $B_{k}(\zeta)$ are holomorphic at $\zeta=-\zeta_{j}^{*-1},-\alpha_{j}^{*-1},-\beta_{j}^{*-1}$.

Proof of Lemma. Substituting $\tilde{J}=X(\zeta) J X^{\top}\left(-\zeta^{*-1}\right)^{\prime}$ into $B_{k}(\zeta)$, we gret

$$
B_{k}(\zeta) \tilde{J}=D_{k} X(\zeta) \cdot J X\left(-\zeta^{*-1}\right)^{\prime}+\zeta^{-1} X(\zeta) J_{\bar{k}} X\left(-\zeta^{*-1}\right)^{\prime},
$$

where $J_{\overline{1}}=\partial_{\bar{z}} J$ and $J_{\overline{2}}=\partial_{\bar{y}} J$. On the other hand,

$$
\begin{aligned}
D_{k} \tilde{J}= & D_{k} X(\zeta) \cdot J X\left(-\zeta^{*-1}\right)^{\dagger}+X(\zeta) D_{k} J \cdot X\left(-\zeta^{*-1}\right)^{\dagger} \\
& +X(\zeta) J D_{k} X\left(-\zeta^{*-1}\right)^{\dagger}
\end{aligned}
$$

Hence we obtain

$(6.16)_{k} \quad B_{k}(\zeta) \tilde{J}=D_{k} \tilde{J}-X(\zeta) J_{k} X\left(-\zeta^{*-1}\right)^{\dagger}-X(\zeta) J D_{k} X\left(-\zeta^{*-1}\right)^{\dagger}$,

where $J_{1}=\partial_{y} J, J_{2}=-\partial_{z} J$. From the assumption $B_{k}(\zeta) \tilde{J}$ is holomorphic at $\zeta=\zeta_{j}, \alpha_{j}, \beta_{j}$. We take the hermitian conjugate of $(6.16)_{1}$ and replace $\zeta$ by $-\zeta^{*-1}$. Noting $J=J^{\dagger}$, we see that

$$
D_{1\left(-\zeta^{*-1}\right)}^{*} X(\zeta) \cdot J X\left(-\zeta^{*-1}\right)^{\prime}+X(\zeta) J_{\bar{y}} X\left(-\zeta^{*-1}\right)^{\dagger}
$$

is holomorphic at $\zeta=-\zeta_{j}^{*-1},-\alpha_{j}^{*-1},-\beta_{j}^{*-1}$. Here

$$
D_{1\left(-\zeta^{+-1}\right)}^{*}=\left.\left(\zeta^{*-1} \partial_{z}+\partial_{\bar{y}}\right)\right|_{\zeta \rightarrow-\zeta^{\prime-1}}=\zeta D_{2} .
$$

Hence $B_{2}(\zeta) \tilde{J},(6.15)_{2}$, is holomorphic at $\zeta=-\zeta_{j}^{*-1},-\alpha_{j}^{*-1},-\beta_{j}^{*-1}$. By the same manner, we can prove the regularity of $B_{1}(\zeta) \tilde{J},(6.15)_{1}$, at $\zeta=-\zeta_{j}^{*-1},-\alpha_{j}^{*-1},-\beta_{j}^{*-1}$. This completes the proof. Q.E.D. 
By Lemma 6.3, each $B_{k}(\zeta)$ has a simple pole at $\zeta=0$ and takes the form

$$
B_{k}(\zeta)=\zeta^{-1} \tilde{A}_{k}
$$

This completes the proof of Theorem 6.2.

Q.E.D.

Finally we discuss the positivity of $\tilde{J}$ for $n=2$. By Theorem 6.2, we have

$$
\operatorname{det} X(0)=(-)^{N_{1}+N_{2}+N_{3}} \prod_{j=1}^{N_{1}}\left|\zeta_{j}\right|^{-2} \prod_{j=1}^{N_{2}}\left|\alpha_{j}\right|^{-2} \prod_{j=1}^{N_{3}}\left|\beta_{j}\right|^{-2}
$$

Due to this, it is seen that $\operatorname{det} J$ and $\operatorname{det} \widetilde{J}$ are of the same sign when $N_{1}+N_{2}+N_{3}$ is even, but they are not so when $N_{1}+N_{2}+N_{3}$ is odd. Thus, as the known Bäcklund transformation [5], [13], the odd transformation $\left(N_{1}+N_{2}+N_{3}\right.$ : odd) violates the positivity of solutions. The authors [17] derived a hermitian solution of the $\mathrm{SL}(2, \boldsymbol{C})$ anti-self-dual equation as an application of the Bäcklund transformation (6.4).

In this paper we have shown that the Riemann-Hilbert problem is useful to study the (anti)-self-dual gauge fields. We emphasize that it is a very important and interesting problem in the furture to investigate other nonlinear equations on four or higher-dimensional spaces by using the technique of the Riemann-Hilbert problem.

\section{References}

[1] Atiyah, M. F. and Ward, R. S., Instantons and algebraic geometry, Comm. Math. Phys., 55 (1977), 117-124.

[2] Belavin, A. A. and Zakharov, V. E., Yang-Mills equations as inverse scattering problem, Phys. Lett., 73B (1978), 53-57.

[3] Belinsky, V. A. and Zakharov, V. E., Stationary gravitational solitons with axial symmetry, Soviet Phys. JETP, 48 (1978), 985-994.

[4] Chau, L. L., Ge, M. L. and Wu, Y. S., Kac-Moody algebra in the self-dual YangMills equation, Phys. Rev., D25 (1982), 1086-1094.

[5] Corrigan, E. F., Fairlie, D. B., Yates, R. G. and Goddard, P., The construction of self-dual solutions to $S U(2)$ gauge theory, Comm. Math. Phys., 58 (1978), 223240.

[6] Devchand, C. and Fairlie, D. B., A generating function for hidden symmetries of chiral model, Nuclear Phys., B194 (1982), 232-236.

[7] Dolan, L., Kac-Moody algebra is hidden symmetry of chiral models, Phys. Rev. Lett., 47 (1981), 1371-1374.

[8] Forgács, P., Harváth, Z. and Palla, L., Towards complete integrability of the selfduality equations, Phys. Rev., D23 (1981), 1876-1879. 
[9] Gantmacher, F. R., The theory of matrices, 1, Chelsea, 1959.

[10] Hauser, I. and Ernst, F. J., Integral equation method for effecting Kinnersley-Chitre transformations, Phys. Rev., D20 (1979), 362-369, A homogeneous Hilbert problem for the Kinnersley-Chitre transformations, J. Math. Phys., 21 (1980), 1126-1140.

[11] Kinnersley, W., Symmetries of the stationary Einstein-Maxwell field equations, I., J. Math. Phys., 18 (1977), 1529-1537.

Kinnersley, W. and Chitre, D. M., Symmetries of the stationary Einstein-Maxwell field equations, II., J. Math. Phys., 18 (1977), 1538-1542, III., ibid. 19 (1978), 19261931, IV. Transformation which preserve asymptotic flatness, ibid. 2037-2042.

[12] Pohlmeyer, K., On the Lagrangian theory of anti-self-dual fields in four-dimensional Euclidean space, Comm. Math. Phys., 72 (1980), 37-47.

[13] Prasad, M. K., Sinha, A. and Wang, L. L. C., Phys. Rev. Lett., 43 (1979), 750-753.

[14] Prasad, M. K., Instanton and monopoles in Yang-Mills gauge field theories, Physica, 1D (1980), 167-189.

[15] Ueno, K., Infinite dimensional Lie algebras acting on chiral fields and the RiemannHilbert problem, Publ. RIMS, Kyoto Univ., 19 (1983), 59-82.

[16] Ueno, K. and Nakamura, Y., Transformation theory for anti-self-dual equations and the Riemann-Hilbert problem, Phys. Lett., 109B (1982), 273-278.

[17] - Infinite dimensional Lie algebras and transformation theories for nonlinear field equations, Proceedings of RIMS symposium on non-linear integrable systems-Classical theory and quantum theory, Kyoto, Japan 1981, World Scientific 1983.

[18] - The hidden symmetry of chiral fields and the Riemann-Hilbert problem, Phys. Lett., 117B (1982), 208-212.

[19] Ward, R. S., On self-dual gauge fields, Phys. Lett., 61A (1977), 81-82.

[20] Yang, C. N., Construction of self-duality for $S U(2)$ gauge fields on Euclidean fourdimensional space, Phys. Rev. Lett., 38 (1977), 1377-1379.

[21] Zakharov, V. E. and Mikhailov, A. V., Relativistically invariant two-dimensional models of field theory which are integrable by means of the inverse scattering problem method, Soviet Phys. JETP, 47 (1978), 1017-1027. 
